\title{
Managing Chronic Urticaria: Quo Vadis?
}

This comprehensive and detailed review by Petkova and Staevska puts chronic urticaria under the spotlight. As the title suggests, the ongoing management of this commonly diagnosed skin disease must be carefully monitored to establish effective treatment plans for patients. The article expertly discusses our current position and guidelines for classification, diagnosis, and management of chronic urticaria including therapeutic options currently under investigation.

\section{Prof Des Tobin}

University College Dublin
Authors:
Elena Petkova, *Maria Staevska
Department of Allergy, Medical University of Sofia, Sofia, Bulgaria
*Correspondence to mstaevska@medfac.mu-sofia.bg
Disclosure:
The authors have declared no conflicts of interest.
Received:
03.02 .20
Accepted:
01.04 .20
Keywords:
Biomarkers, chronic urticaria (CU), classification, diagnosis, management, therapy response.
Citation:
EMJ Dermatol. 2020;8[1]:66-74.

\begin{abstract}
Chronic urticaria (CU) is one of the most commonly diagnosed skin conditions. $\mathrm{CU}$ is characterised by the presence of recurrent wheals and/or angioedema and intense pruritus persisting for at least 6 weeks. Subtypes of CU include chronic spontaneous urticaria and chronic inducible urticaria. Following diagnosis, adequate trigger identification and appropriate treatment can significantly reduce disease activity and improve the patient's quality of life and disease outcomes. Current guidelines recommend a stepwise approach in the management of $\mathrm{CU}$, including non-sedating oral antihistamines, administered in up to four times the conventional dose, the monoclonal antibody omalizumab (antiIgE), and eventually cyclosporine as an add-on therapy for patients with antihistamine-refractory CU. Potential disease-related biomarkers are needed to predict the therapeutic response that would lead to establishment of personalised regimens and treatment plans. This paper reviews the current perspectives and guidelines for classification, diagnosis, and management of CU.
\end{abstract}

\section{INTRODUCTION}

Urticaria is one of the most common skin diseases, and one of the most common reasons for a general practitioner, paediatrician, dermatologist, allergist consultation, or emergency room visit.

It is estimated that up to $25 \%$ of the American population experience at least one episode of urticaria during their lifetime. ${ }^{2}$ In $50 \%$ of all patients, urticaria and angioedema occur simultaneously: $40 \%$ only have hives, and $10 \%$ present with isolated angioedema. ${ }^{3}$ A study 
by Baptist and Baldwin ${ }^{4}$ showed that general practitioners were likely to refer patients with atopic dermatitis to dermatologists and those with chronic urticaria (CU) to allergists. ${ }^{4}$

\section{DEFINITION}

According to the 2018 International Consensus Guidelines for the Diagnosis and Treatment of Urticaria, urticaria is characterised by the sudden appearance of wheals, angioedema, or both. ${ }^{5}$ Wheals or hives are characterised by central oedema of variable size, almost always surrounded by a reflex erythema, which disappears with vitropression. They have a transient nature and the skin returns to normal usually within 30 minutes to 24 hours. Patients usually report an itching or burning sensation. Itching is relieved by rubbing the skin rather than by scratching it. Excoriations are therefore not common in urticaria, which helps to differentiate it from atopic dermatitis, typically characterised by severe skin excoriations. Angioedema is characterised by sudden, marked, erythematous, or pale swelling of the underlying dermis and subcutaneous tissue or mucous membranes that can be painful rather than itchy. This can have a slower resolution of symptoms compared to papules, which would take up to 72 hours. $^{5}$

\section{CLASSIFICATION}

Urticaria is generally classified as acute or chronic. Acute urticaria usually resolves within 6 weeks. An external cause can be identified in approximately $50 \%$ of all cases: viral infections are the most common causes and less common triggers include specific drugs or food. The cause may not be identified in the remaining $50 \%$ of patients. ${ }^{6} \mathrm{CU}$ and angioedema are defined as daily or almost daily symptoms for $>6$ weeks. The cases of intermittent urticaria, recurrent episodes of urticaria that last for a few minutes or several days, were also included in this definition. ${ }^{7}$ In turn, $\mathrm{CU}$ can be classified as spontaneous and inducible. The term 'spontaneous' underlines the spontaneous nature of rashes and oedema as opposed to inducible urticaria, in which rashes are triggered by specific factors, for example, symptomatic dermographism, cold urticaria, delayed pressure urticaria, solar urticaria, heat urticaria, vibratory angioedema, cholinergic urticaria, contact urticaria, and aquagenic urticaria. In spontaneous urticaria, rashes usually develop spontaneously; however, in some patients the condition can be aggravated by certain triggers, such as stress, viral infections, and nonsteroidal anti-inflammatory drugs (NSAID). It is important to distinguish recurrent chronic intermittent urticaria from the repeated episodes of acute urticaria. For example, if a patient suffers multiple episodes of antibiotic-induced acute urticaria, they are considered separate episodes of acute urticaria as a manifestation of a drug allergy; in the case of multiple episodes of NSAID-induced urticaria the episodes are more likely to be a presentation of chronic intermittent spontaneous urticaria, which is exacerbated by NSAID via a pseudoallergic mechanism. Often, the administration of NSAID overlaps with viral infections which makes it difficult to determine the risk factor for exacerbations. Intermittent $\mathrm{CU}$ triggered by viral infections is more common in children. Importantly, two or more urticaria subtypes, for example, chronic spontaneous urticaria (CSU) and symptomatic dermographism/delayed pressure urticaria, can coexist in the same patient.

Following the natural course of the disease, remission of CSU is achieved in 50\% of patients within 6 months and 20\% of them become asymptomatic at Year 3 and another 20\% at Year 5 of disease onset. It is estimated that $2 \%$ of all patients will achieve remission after 25 years. However, at least one-half of patients will relapse at least once after remission. ${ }^{8}$ Recent data shows somewhat similar results for remission rates: approximately 25\% after 3 months; 50\% after 1 year; approximately $80 \%$ after 3 years; and approximately $90 \%$ after 5 years. ${ }^{9}$

Despite similar terminology or presentation, rash and/or oedema, urticaria pigmentosa (cutaneous mastocytosis), urticarial vasculitis, familial cold urticaria, and bradykinin-mediated angioedema (for example, hereditary and acquired angioedema or angiotensin-converting enzyme inhibitor-related angioedema) are not urticaria subtypes; however, they should be considered in the differential diagnosis of urticaria. CU and other urticaria subtypes can be signs and symptoms of other conditions or syndromes. ${ }^{5}$ 
Inducible urticaria:

$<1$ hour

Contact urticaria: 1-2 hours

Spontaneous and delayed pressure urticaria: 2-24 hours

Urticarial vasculitis: 1-7 days

\section{Figure 1: What is the average wheal duration?}

The duration of wheals is an important factor in the initial diagnosis of urticaria.

\section{DIAGNOSIS AND DIFFERENTIAL DIAGNOSIS}

The presence of extensive skin lesions is generally associated with a disease with a more severe course which is more difficult to treat. Skin lesion colour may provide useful information. The histamine-induced papules are light-coloured with surrounding pink erythema because of skin vessel dilation. In contrast, dark red or purple papules are signs of more intense vascular damage and impaired vessel integrity, which are typical features of urticarial vasculitis. ${ }^{5}$ The size of the lesions and the affected area is associated with the disease severity, but not with the specific type of urticaria or angioedema. The duration of lesions is a useful guide for differentiating between the various conditions as they may have overlapping features (Figure 1)..$^{10}$

\section{DIAGNOSIS}

Acute urticaria is a self-limiting condition and therefore the International Consensus guidelines do not recommend extensive diagnostic procedures. $^{5}$ Diagnostic evaluation can be useful in the cases of food allergy and IgEmediated NSAID hypersensitivity (pyrazolones, acetaminophen, ketorolac, nimesulide). ${ }^{11}$ The three main goals of the diagnostic workup in $\mathrm{CU}$ are to rule out differential diagnoses (diagnostic algorithm and main differential diagnoses are shown in Figure 2);:12 to evaluate the disease activity and its impact on control and quality of life by using several validated tests for disease activity assessment including urticaria activity score (UAS), angioedema activity score (AAS), urticaria and angioedema quality of life questionnaires (CU-Q2oL, AEQoL), and the urticaria control test (UCT); and to identify risk factors and exacerbation triggers, as well as aetiology, if possible. Type 1 hypersensitivity reactions are rarely recognised as causes of chronic persistent urticaria but can be considered in some cases. For example, in food-dependent, exercise-induced anaphylaxis, one should take into consideration both allergic and nonallergic food sensitivity, especially Type 1 allergic reaction to cereals and gliadin, as well as nonspecific reactions to alcohol (alcohol intolerance). CU may be triggered by an underlying persistent infection caused by Helicobacter pylori, Streptococci, Staphylococci, or Yersinia. ${ }^{13}$ The incidence and impact of infectious diseases may vary considerably between different patient groups and regions. For example, viral hepatitis is a common cause of $\mathrm{CU}$ in Southern European countries but is rarely associated with the condition in Northern Europe. 


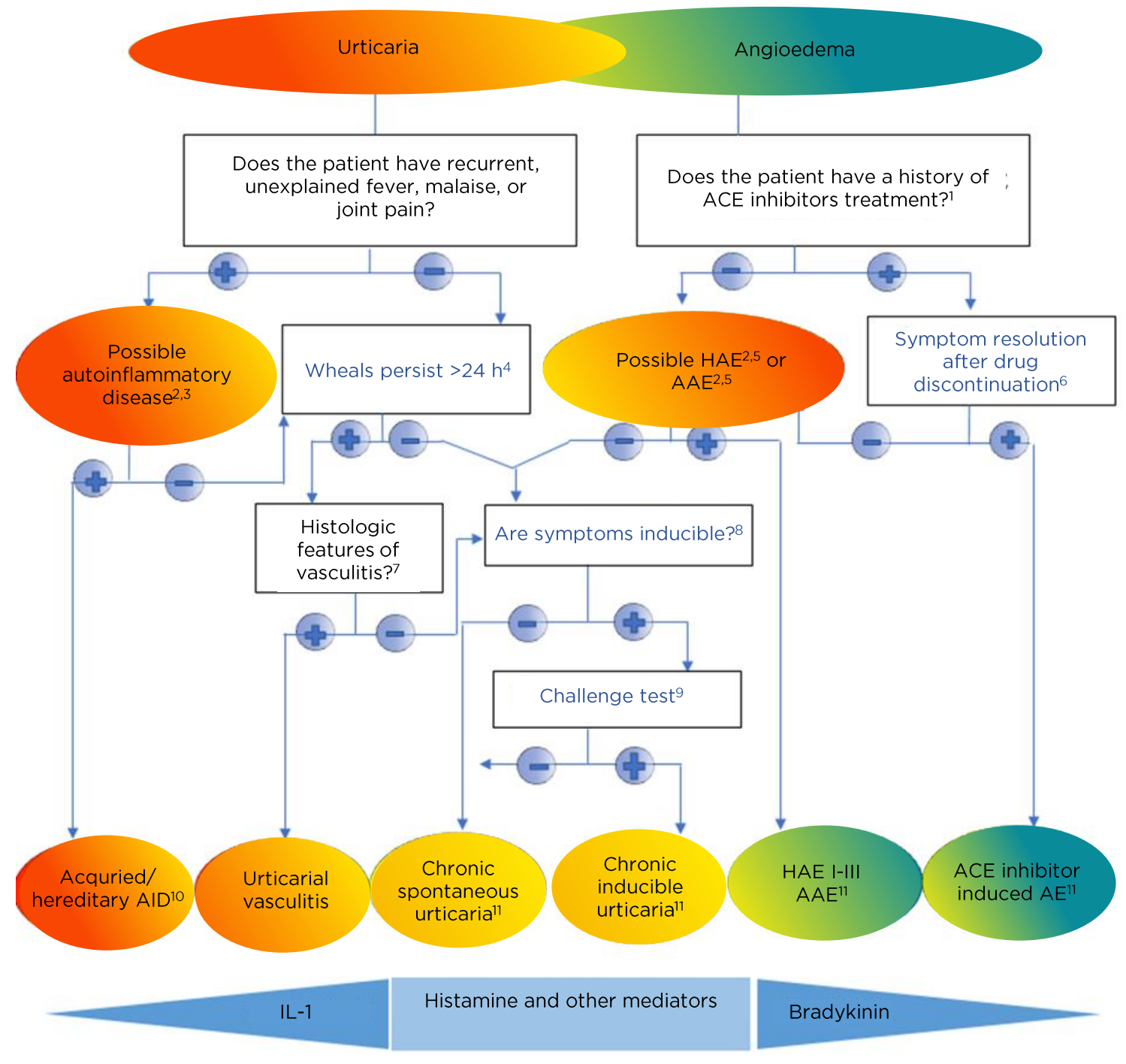

Figure 2: Diagnostic algorithm for urticaria, angioedema or both.

1Apart from ACE-inhibitors, other renin inhibitors and sartans can also cause angioedema but much less frequently. ${ }^{2}$ Patients should be asked for a detailed family history and age of disease onset. ${ }^{3}$ Tests for elevated inflammatory markers (C-reactive protein, erythrocyte sedimentation rate), tests for paraproteinaemia in adults, look for signs of neutrophil infiltration in skin biopsy; gene mutation analysis for hereditary periodic fever syndromes (for example, cryopyrin-associated periodic syndrome), if strongly suspected. ${ }^{4}$ Patients should be asked: "For how long does each wheal last?" " Test for complement C4, C1-INH levels, and function; in addition, test for Clq and C1-INH antibodies, if AAE is suspected; gene mutation analysis, if tests are normal but history of hereditary angioedema. ${ }^{6}$ If no remission is achieved after 6 months of ACE-inhibitor discontinuation, C1-inhibitor should be tested. ${ }^{7}$ Does the skin biopsy show small vessels in the papillary and reticular dermis damage and/or fibrinoid deposits in perivascular and interstitial areas suggestive of urticarial vasculitis? ${ }^{8}$ Patients should be asked: "Can you trigger your wheals? Can you bring out your wheals?" In patients with a history of inducible urticaria standardised provocation testing according to International Consensus recommendations should be performed. ${ }^{10}$ Acquired autoinflammatory syndromes include Schnitzler's syndrome, systemic juvenile idiopathic arthritis, and adult-onset Still's disease; hereditary autoinflammatory syndromes include cryopyrin-associated periodic syndromes such as familial cold autoinflammatory syndrome, Muckle-Wells syndrome, neonatal-onset multisystem inflammatory disease, and more rarely hyper-IgD syndrome, and TNFa-associated periodic syndrome. "In some rare cases, recurrent angioedema is neither mast cell-mediated nor bradykinin-mediated, and the underlying pathomechanisms remain unknown. These rare cases are referred to as "idiopathic angioedema" by some authors.

AAE: acquired angioedema because of C1-inhibitor deficiency; ACEi: angiotensin-converting enzyme inhibitor; AE: adverse event; AID: autoinflammatory disease; HAE: hereditary angioedema.

Adapted from Zuberbier et al.5; Magerl et al. ${ }^{12}$ 
Infestation with Anisakis simplex, a sea fish nematode, may be an important cause of anaphylaxis in regions with raw fish consumption. ${ }^{14}$ The incidence of dental and ear, nose, and throat infections appears to vary between patient groups. In general, laboratory tests are rarely outside the normal range and very rarely influence diagnosis and management of the disease. ${ }^{15}$

Acetylsalicylic acid (aspirin) and other NSAID inhibit cyclooxygenase (COX)-1 and inducible COX-2, thus diverting arachidonic acid metabolism towards the 5-lipoxygenase metabolic pathway in certain cells, especially eosinophils. ${ }^{16}$ This modulation is associated with overproduction of cysteinyl-leukotrienes LTC4, D4, and E4 which results in vasodilation and oedema. There is a known cross-sensitivity between different nonselective NSAID in affected individuals that depends on their pharmacological ability to inhibit COX rather than on their chemical structure. ${ }^{17}$

Aspirin and NSAID can cause both acute urticaria and exacerbation of pre-existing chronic spontaneous, but not physical, urticaria. The incidence of intolerance is $0.3 \%$ in the general population, ${ }^{18}$ whereas aspirin-induced CSU exacerbations have been reported in $20-40 \%$ of all patients. ${ }^{18,19}$ At least $22 \%$ of patients with CSU visit the emergency room or hospital for disease exacerbation because of aspirin intake, and not because of physical urticaria. ${ }^{18}$ Furthermore, aspirin-induced acute urticaria is a risk factor for CSU development."

Currently, the only widely available test for screening for autoantibodies against the $\lg \mathrm{E}$ receptor is the autologous serum skin test. This is a nonspecific test that assesses the presence of serum histamine releasing factors of all types, not just autoantibodies. This test should be performed with caution to minimise potential risks of accidental infection in case the patient is injected with unknown serum by mistake. In some specialised centres, more specific laboratory tests for in vitro histamine release from basophils, using a basophil histamine release assay (BHRA) evaluation or the basophil activation test, are also available and could be used for autoantibody search.

\section{MANAGEMENT}

The recent international consensus EAACl/ GA²LEN/EDF/WAO guideline for the definition, classification, diagnosis, and management of urticaria $2018^{5}$ includes nonpharmacological approaches such as identification and elimination of underlying causes, triggering factor avoidance, and inducing tolerance, and pharmacological treatment.

\section{Identification and Elimination of Underlying Causes}

Spontaneous remission of $\mathrm{CU}$ can occur at any time in the disease course; therefore, it can be very difficult to assess the effect of elimination of a suspected cause or trigger. For example, concomitant infection may be a cause, aggravating factor, or unrelated. Remission and elimination of the suspected cause can also occur coincidentally. Underlying immunological mechanisms, autoimmunity and autoallergy associated with the persistent disease also may not be eliminated.

\section{Triggering Factor Avoidance}

NSAID can be a causative or aggravating factor in approximately $20-40 \%$ of patients with CU and in $17 \%$ of Bulgarian patients (Staevska, unpublished data). Elimination of these drugs and use of non-COX-1 agents, namely specific COX2 inhibitors and paracetamol (acetaminophen), is recommended in these patients. Patients with inducible urticaria should avoid known eliciting factors such as lifting heavy objects, intense pressure in delayed pressure urticaria, or friction in dermographism. Eradication of infectious agents and treatment of inflammatory processes are recommended, although studies show conflicting results about their effect on the natural course of the disease. Reduction of physical and emotional stress is beneficial as there is some evidence that disease activity and severity are correlated with stress levels. ${ }^{20}$ Plasmapheresis of functional autoantibodies may be recommended in some severely affected patients, but this treatment is neither established nor widely available. 


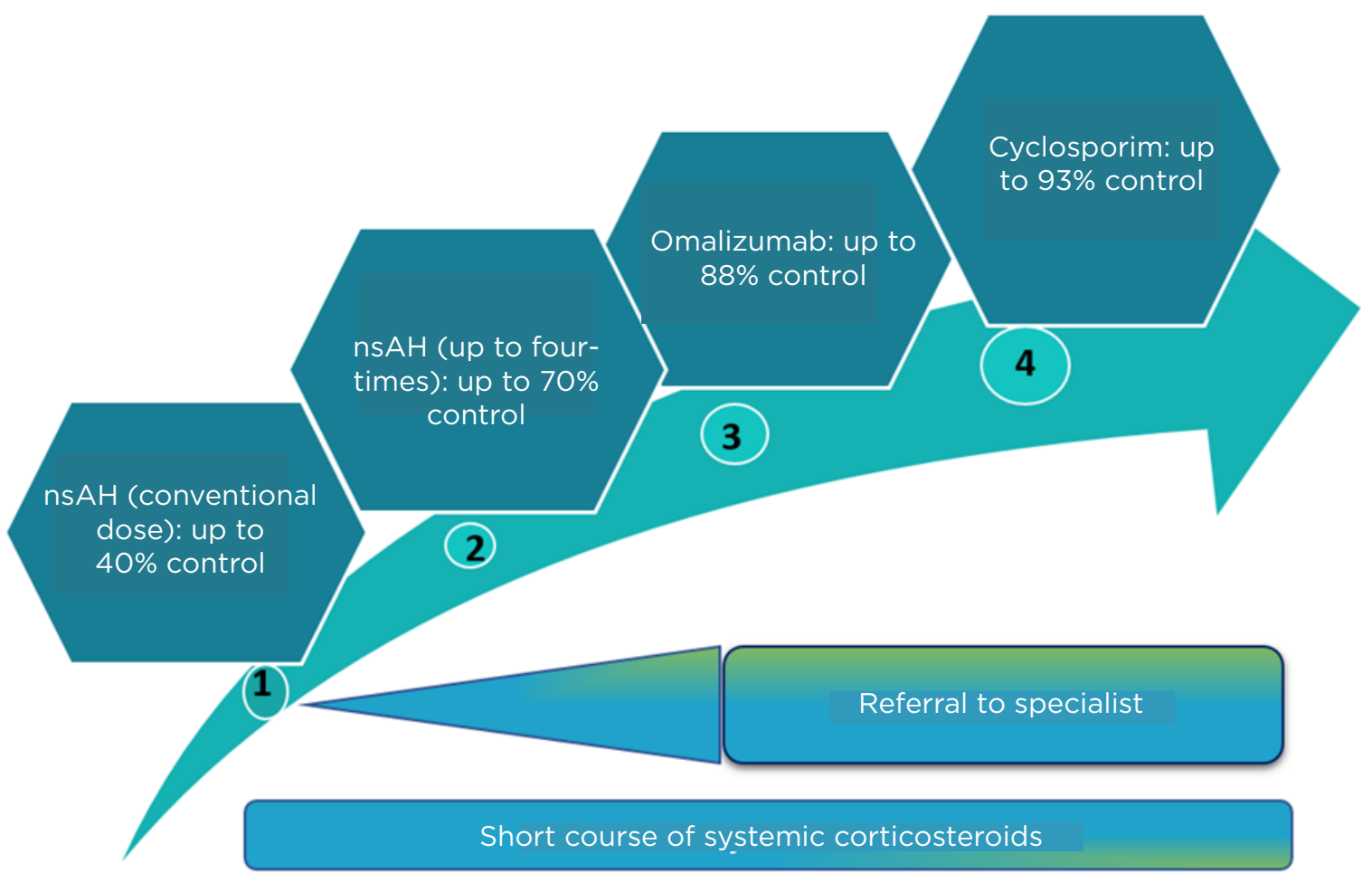

Figure 3: Rates of control, achieved with first-, second-, third-, and fourth-line treatment, according to the 2018 International Consensus guidelines.

nsAH: nonsedating antihistamines.

Adapted from Zuberbier et al.5; Kocatürk et al. ${ }^{22}$

Dietary restrictions are only recommended in the case of IgE-mediated food allergy. Avoidance of histamine-, pseudoallergen-rich foods, or foods containing salicylates has been proposed but is a controversial measure because there is a lack of 'good' evidence from randomised controlled trials to draw conclusive evidence about the diet's effectiveness. ${ }^{21}$

\section{Inducing Tolerance}

Inducing tolerance can be achieved in some subtypes of inducible urticaria, such as cold urticaria, cholinergic urticaria, and solar urticaria. However, tolerance only lasts for a few days and its maintenance is often not accepted by patients; for example, in cases of cold urticaria where daily cold showers are needed to achieve tolerance.

\section{Pharmacological Treatment}

Treatment recommendations were developed using the Grading of Recommendations Assessment, Development and Evaluation
(GRADE) system (Figure 3). ${ }^{5}$ A structured consensus process was used by the International Consensus Working group to review evidence and discuss and agree upon recommendations. The main goal of the pharmacological treatment is to achieve complete symptom relief.

Another general principle in pharmacotherapy is to use as much as necessary as little as possible and thus the treatment may vary in the disease course. First-line treatment includes modern second-generation $\mathrm{H} 1$-antihistamines in licensed doses. The International Consensus guidelines recommend that modern secondgeneration antihistamines should be considered as the first-line symptomatic treatment for urticaria because of their good safety profile. The use of first-generation antihistamines is not recommended. Experts advise that antihistamines should be taken daily, or regularly, rather than only when symptoms occur, or as needed. It is estimated that approximately $40 \%$ of all patients achieve control with this treatment. ${ }^{6}$ 
Second-line treatment includes up-dosing to four-fold the conventional dose of modern second-generation H1-antihistamines. Up-dosing second-generation $\mathrm{H} 1$-antihistamines to fourfold is recommended in patients with $\mathrm{CU}$ with inadequate control or intolerable symptoms within 2-4 weeks of treatment or earlier. Many studies demonstrate the benefit and safety of a higher dosage of second-generation antihistamines. ${ }^{23}$ Patients with urticaria who do not respond to up-dosing of $\mathrm{H} 1$-antihistamines to four-fold the licensed dose are not recommended to receive further up-dosing. Some experts advise against using different $\mathrm{H} 1$-antihistamines at the same time, although this is an expert opinion and scientific evidence is lacking. Although some authors expect achievement of disease control with antihistamines in $<50 \%$ of all patients ${ }^{24}$ others report achieving control in up to $70 \%$ of patient with CU.23,24 Older guidelines recommend adding a first-generation antihistamine at night;:25 however, the International Consensus guidelines recommend against the use of these sedating antihistamines for the routine management of $\mathrm{CU}$, which is also supported by the authors' own experiences. ${ }^{26}$

More recently, several biomarkers related to the disease prognosis and therapeutic response have been described in CSU. ${ }^{27}$ Asero $^{28}$ demonstrated that higher levels of D-dimer are associated with insufficient clinical response to antihistamines. Kolkhir et al. ${ }^{29}$ confirmed these results and showed that not only measurements of D-dimer, but also high levels of fibrinogen, C-reactive protein, and erythrocyte sedimentation rate should be considered predictors of poor response to antihistamines. Other biomarkers for antihistamine-resistant CSU could be increased complement C5a levels, higher disease activity, longer duration of wheals, and higher autologous serum skin test positivity. ${ }^{30}$

Third-line treatment includes adding on omalizumab to nonsedating second-generation H1-antihistamines. Many studies have demonstrated that omalizumab (anti-IgE) is very effective and safe in the treatment of CSU and inducible urticaria and is currently licensed for these indications. In CSU, omalizumab prevents angioedema development, significantly improves quality of life, and is suitable for long-term treatment. The recommended dose is $300 \mathrm{mg}$ every 4 weeks. Dosing is independent of total serum IgE. Add-on treatment with omalizumab is effective in $65-70 \%$ of patients who are unresponsive to high doses of $\mathrm{H} 1$-antihistamines and $35-40 \%$ of patients are able to achieve complete symptom control. ${ }^{31}$ Thus, based on the total patient population, it can be estimated that control can be achieved with antihistamines and omalizumab in $80-85 \%$ of patients with urticaria, ${ }^{32}$ though one study has suggested this could be up to $88 \%{ }^{22}$

Several biomarkers related to treatment with omalizumab have been proposed. A recent study showed that lower baseline levels of IL31 were associated with satisfactory clinical response. ${ }^{33}$ A recent Spanish study demonstrated that lower baseline levels of basophil highaffinity IgE receptor (FcERI) expression was associated with insufficient clinical response to omalizumab. ${ }^{34}$ Ertas et al. ${ }^{35}$ found that clinical response to omalizumab can be predicted by total serum IgE levels and their change during treatment, particularly by Week 4/baseline ratio of total IgE (lower baseline levels and a lesser increase after start of treatment predict insufficient clinical response to omalizumab). ${ }^{35}$ More recently, Riccardo et al. ${ }^{36}$ added that this biomarker could be more specific in nonatopic nonresponders with low levels of IgE than in atopic nonresponders and speculated that this finding could be considered as indirect evidence for pathogenetic role of autoreactive IgE. ${ }^{36}$ Additionally, BHRA and autologous serum skin test positivity have been recently proposed as predictors for slow therapeutic response to omalizumab, whereas increased IgE levels seem to be associated with faster relapse. ${ }^{37,38}$

Fourth-line treatment includes adding cyclosporin A to nonsedating second-generation $\mathrm{H} 1$-antihistamines. Cyclosporine $A$ has a moderate, direct effect on histamine release. Efficacy of cyclosporine $A$ in combination with a second-generation $\mathrm{H} 1$-antihistamine has been demonstrated in two placebo-controlled trials including $129 \mathrm{CU}$ patients (45 with placebo), ${ }^{39}$ but it cannot be recommended as standard treatment because of the higher risk of adverse effects. Cyclosporine $A$ is not licensed for the treatment of urticaria and its off-label use in urticaria is recommended for patients with severe refractory disease to combinations 
of antihistamine and omalizumab only. The cyclosporine dose suggested for urticaria is $2-4 \mathrm{mg} / \mathrm{kg} / \mathrm{day}^{39}$ It should be noted that cyclosporine $\mathrm{A}$ has a far better risk/benefit ratio compared with long-term use of corticosteroids. Cyclosporine A increases the success rate of CU treatment by up to $93 \%{ }^{22,32}$

Recent studies show that baseline levels of D-dimer and BHRA are linked to response to cyclosporine. Asero ${ }^{40}$ found that lower D-dimer levels are associated with satisfactory clinical response to cyclosporine, which suggests that D-dimer levels could be a useful tool to predict and monitor clinical response to cyclosporine. Furthermore, two independent studies demonstrated that BHRA positivity is associated with satisfactory clinical response to cyclosporine. ${ }^{41,42}$

Leukotriene receptor antagonists and $\mathrm{H} 2-$ antihistamines are no longer recommended by the International Consensus guidelines due to low levels of evidence for their efficacy in urticaria. For acute urticaria and acute exacerbations of $\mathrm{CU}$, a short course of oral corticosteroids may be used to reduce disease duration and activity. Treatment with systemic corticosteroids should be limited to a maximum of up to 10 days in doses between 20 and $50 \mathrm{mg} /$ day (Figure 3). ${ }^{5}$ Tacrolimus, mycophenolate, sulfones (dapsone and sulfasalazine), and hydroxychloroquine were also tested for the treatment of CSU but evidence in support of their use is limited.

\section{CONCLUSION}

In recent years, the use of high dose nonsedating antihistamines, and especially omalizumab, has been considered a revolution in the treatment of $\mathrm{CU}$ but unfortunately the price (lack of reimbursement in some countries) limits the use of this highly effective biological treatment. Other biologics are currently under investigation for the treatment of refractory CSU. These include the high-affinity anti-lgE monoclonal antibody, ligelizumab; the anti-IL-5 monoclonal antibody, mepolizumab; the anti-IL-5 receptor a monoclonal antibody, benralizumab; the antiIL-4 and IL-13 monoclonal antibody, dupilumab; antisialic acid-binding Ig-like lectin-8 drugs such as spleen tyrosine kinase inhibitors; oral treatments such as LOU064, a Bruton's tyrosine kinase selective inhibitor; ${ }^{43}$ antagonists of prostaglandin D2 receptor 2; and IL-1 inhibitors. ${ }^{44}$

\section{References}

1. Maurer $M$ et al. Unmet clinical needs in chronic spontaneous urticaria. A $\mathrm{GA}^{2} \mathrm{LEN}$ task force report. Allergy. 2010:66(3);317-30.

2. Henderson et al. Allergists and dermatologists have far more expertise in caring for patients with urticaria than other specialists. J Am Acad Dermatol. 2000;43(6):1084-91.

3. Champion $\mathrm{RH}$ et al. Urticaria and angio-oedema: a review of 554 patients. Br J Der-matol. 1969;81(8):588-97.

4. Baptist AP, Baldwin JL. Physician attitudes, opinions, and referral patterns: compari-sons of those who have and have not taken an allergy/ immunology rotation. Ann Allergy Asthma Immunol. 2004;93(3):227-31.

5. Zuberbier $\mathrm{T}$ et al. The EAACl/ GA2LEN/EDF/WAO guideline for the definition, classification, diagnosis and management of urticaria. Allergy. 2018;73(7):1393-1414.

6. Kulthanan $\mathrm{K}$ et al. Acute urticaria: etiologies, clinical course and quality of life. Asian Pac J Allergy Immunol. 2008;26(1):1-9.
7. Powell RJ et al. BSACI guidelines for the management of chronic urticaria and an-gioedema. Clin Exp Allergy. 2015;45(3):547-65.

8. Beltrani VS. An overview of chronic urticaria. Clin Rev Allergy Immunol. 2002;23(2):147-69.

9. Eun SJ et al. Natural course of new-onset urticaria: results of a 10-year follow-up, nationwide, population-based study. Allergol Int. 2019;68(1):52-8.

10. Grattan CE et al. Chronic urticaria. J Am Acad Dermatol. 2002;46(5) :645-57.

11. Asero R. Intolerance to nonsteroidal anti-inflammatory drugs might precede by years the onset of chronic urticaria. J Allergy Clin Immunol. 2003;111(5):1095-8.

12. Magerl $M$ et al. The definition, diagnostic testing, and management of chronic in-ducible urticarias the EAACI/GA(2) LEN/EDF/UNEV consensus recommenda-tions 2016 update and revision. Allergy. 2016;71(6):780-802.
13. Wedi B et al. Chronic urticaria and infections. Curr Opin Allergy Clin Immunol. 2004;4(5):387-96.

14. Foti $\mathrm{C}$ et al. Acute allergic reactions to Anisakis simplex after ingestion of ancho-vies. Acta Derm Venereol. 2002;82(2):121-3.

15. Tarbox $\mathrm{J}$ et al. Utility of routine laboratory testing in management of chronic urticar-ia/angioedema. Ann Allergy Asthma Immunol. 2011;107(3):239-43.

16. Borzova E, Grattan CEH, "Urticaria, angioedema and anaphylaxis," Rich R et al. (eds.), Clinical Immunology: Principles and Practice (2019) $3^{\text {rd }}$ edition, Amsterdam: Elsevier, pp.641-656.

17. Kowalski ML et al. Hypersensitivity to nonsteroidal anti-inflammatory drugs (NSAIDs) - classification, diagnosis and management: review of the EAACI/ENDA\# and GA2LEN/ HANNA*. Allergy. 2011;66:818-29.

18. Grattan CE. Aspirin sensitivity and urticaria. Clin Exp Dermatol. 2003;28(2):123-7. 
19. "Urticaria and Angioedema," Greaves MW, Kaplan A (eds.), Urticaria and Angi-oedema (2004), New York: Marcel Dekker Ltd.

20. Varghese $\mathrm{R}$ et al. Association among stress, hypocortisolism, systemic inflammation, and disease severity in chronic urticaria. Ann Allergy Asthma Immunol. 2016;116(4):344-8.e1.

21. Chiang $\mathrm{HL}$ et al. Which fruits and vegetables should be excluded from a low-salicylate diet? An analysis of salicylic acid in foodstuffs in Taiwan. Int Arch Allergy Immunol. 2018;176(34):198-204

22. Kocatürk E et al. Management of chronic inducible urticaria according to the guide-lines: a prospective controlled study. J Dermatol Sci. 2017;87(1):60-9.

23. Staevska M et al. The effectiveness of levocetirizine and desloratadine in up to 4 times conventional doses in difficult-to-treat urticaria. J Allergy Clin Immunol. 2010:125(3):676-82.

24. A Kaplan. Chronic spontaneous urticaria: pathogenesis and treatment considera-tions. Allergy Asthma Immunol Res. 2017;9(6):477-82.

25. Bernstein $\mathrm{J}$ et al. The diagnosis and management of acute and chronic urticaria: 2014 update. J Allergy Clin Immunol. 2014;133(5):1270-7.

26. Staevska $M$ et al. Night-time sedating $\mathrm{H} 1$-antihistamine increases daytime somno-lence but not treatment efficacy in chronic spontaneous urticaria: a randomized controlled trial. Br J Dermatol. 2014;171(1):148-54.

27. Deza $\mathrm{G}$ et al. Emerging biomarkers and therapeutic pipelines for chronic spontane-ous urticaria. J Allergy Clin Immunol Pract. 2018;6(4):1108-17.

28. R Asero. D-dimer: a biomarker for antihistamine-resistant chronic urticaria. J Allergy Clin Immunol. 2013;132(4):983-6.

29. Kolkhir $P$ et al. CRP, D-dimer fibrinogen and ESR as predictive markers of response to standard doses of levocetirizine in patients with chronic spontaneous urticaria. Eur Ann Allergy Clin Immunol. 2017:49(9):189-92.

30. Huilan $Z$ et al. Features of antihistamine - resistant chronic urticaria and chronic urti-caria during exacerbation. Indian J Dermatol. 2015;60(3):323.

31. Saini SS, Kaplan AP. Chronic spontaneous urticaria: the devil's itch. J Allergy Clin Immunol Pract. 2018:6(4):1097-1106.

32. Kaplan A. Diagnosis, pathogenesis, and treatment of chronic spontaneous urticaria. Allergy and Asthma Proc. 2018;39(3):184-90.

33. Altrichter $\mathrm{S}$ et al. Successful omalizumab treatment in chronic spontaneous urticaria is associated with lowering of serum IL-31 levels. $J$ Eur Acad Dermatol Venereol. 2016;30(3):454-5.

34. Deza G et al. Basophil FceRI expression in chronic spontaneous urticaria: a potential immunological predictor of response to omalizumab therapy. Acta Derm Venereol. 2017;97(6):698-704

35. Ertas R et al. The clinical response to omalizumab in chronic spontaneous urticaria patients is linked to and predicted by lgE levels and their change. Allergy. 2018;73(3):705-12.

36. Riccardo A et al. Total IgE and atopic status in patients with severe chronic sponta-neous urticaria unresponsive to omalizumab treatment. Allergy. 2019;74(8):1561-2.
37. Gericke J et al. Serum autoreactivity predicts time to response to omalizumab ther-apy in chronic spontaneous urticaria. J Allergy Clin Immunol. 2017;139(3):1059-61.

38. Ertas R et al. Increased IgE levels are linked to faster relapse in patients with omali-zumabdiscontinued chronic spontaneous urticaria. J Allergy Clin Immunol. 2017;140(6):1749-51

39. Mitchell S et al. Systematic review of treatments for chronic spontaneous urticaria with inadequate response to licensed first-line treatments. Int Dermatol. 2015;54(9):1088-104.

40. Asero R. Plasma D-dimer levels and clinical response to ciclosporin in severe chron-ic spontaneous urticaria. J Allergy Clin Immunol. 2015;135(5):1401-3

41. Grattan CE et al. Randomized doubleblind study of cyclosporin in chronic 'idio-pathic' urticaria. Br J Dermatol. 2000;143(2):365-72

42. Iqbal $\mathrm{K}$ et al. A positive serum basophil histamine release assay is a marker for ciclo-sporinresponsiveness in patients with chronic spontaneous urticaria. Clin Transl Al-lergy. 2012;2(1):19.

43. Novartis Pharmaceuticals. Dosefinding Study to Evaluate Efficacy and Safety of LOU064 in Patients with CSU Inadequately Controlled by H1-antihistamines. NCTO3926611. https://clinicaltrials.gov/ct2/show/ NCT03926611.

44. Min TK, Saini SS. Emerging therapies in chronic spontaneous urticaria. Allergy Asthma Immunol Res. 2019;11(4):470-81. 